\title{
La resistencia y la resistencia civil: la importancia de la teoría noviolenta*
}

\author{
Resistance, Civil Resistance: the Importance of \\ Nonviolence Theory
}

Daniel Ricardo Martínez Bernal ${ }^{* *}$

Recibido: 27 de agosto de 2014

Aprobado: 26 de enero de 2015

Disponible en línea: 20 de diciembre de 2016

\section{Resumen}

Son muchos los artículos, los textos y las publicaciones que hacen referencia a la resistencia y la resistencia civil, pero muy pocos los que hablan de la noviolencia como uno de sus pilares. Es más, es tal su desconocimiento que noviolencia la escriben indistintamente (no violencia, noviolencia o no-violencia). Por esa razón, el objeto de este artículo es darle un giro a estas ideas, y mostrar cómo la noviolencia es el sustento de las nociones de resistencia y resistencia civil. Gracias a los estudios y las luchas gandhianos es que estos dos conceptos cobraron mayor sentido y en esa medida es que se les puede reconocer como luchas distintas de las militares o bélicas. El fundamento teórico de la noviolencia permite ver tanto la importancia que tiene el factor de lo no armado como la relevancia del estudio de los medios-métodos y fines-estrategias. Tomar como base estas dos variables amplía las

\section{Abstract}

There are many articles, writings and publications related to resistance (in general) and civil resistance, in particular; however, only few of them mention nonviolence as one of its pillars. Such is their lack of recognition that nonviolence can be written indistinctively. The objective of this article is, thus, to challenge these ideas, by illustrating how nonviolence is the root of both resistance and civil resistance. Thanks to Gandhian struggles and studies, these concepts acquired greater importance, highlighting its differentiation from the military or warlike alternatives. Nonviolence's theoretical framework reveals the importance of the 'non-armed' as well as the relevance of the study of means-methods and goals-strategies. Taking these two aspects as a basis broadens the analytical perspective and, mainly, allows for the introduction of values such as 'peace' amidst the constant predominance of

doi:10.11144/Javeriana.papo21-2.rrci

* Artículo de investigación. Este artículo es fruto de la tesis doctoral presentada en el Instituto de Paz y Conflictos de la Universidad de Granada el 12 de enero de 2016.

** Universidad de Granada (Granada, España). Correo electrónico: drmart@correo.ugr.es 
perspectivas de análisis y, sobre todo, permite incluir valores, como el de la paz, en medio de una constante tan predominante como la violencia. Pensar desde el punto de vista de la noviolencia y sus diferentes acepciones facilita entender y conceptualizar las luchas sociales de forma más precisa y sistematizada.

\section{Palabras clave}

resistencia; resistencia civil; noviolencia, medios-métodos y fines-estrategias; Gandhi; resistencia civil noviolenta

\section{Cómo citar este artículo:}

Martínez-Bernal, D. R. (2016). La resistencia yla resistencia civil: la importancia de la teoría noviolenta. Papel Político, 21(2), 343-371. https:// doi.org/10.11144/Javeriana.papo21-2.rrci violence. Thinking in terms of nonviolence, and its different meanings, facilitates a more precise and systematized comprehension and conceptualization of social struggles and civil resistance.

\section{Keywords}

civil resistance; social struggles; nonviolence; means-methods and goals-strategies; Gandhi; nonviolence civil resistance 


\section{Introducción}

Tras hechos tan significativos como las manifestaciones y protestas en Seattle, en 1999, contra la reunión de la Organización Mundial del Comercio (OMC), el fortalecimiento de movimientos y organizaciones sociales con perfiles muy diversos, como los sindicatos en los Estados Unidos, los movimientos indígenas en América Latina, el Ejército Zapatista de Liberación Nacional, los campesinos sin tierra, tanto en Brasil como en otros lugares del mundo, las organizaciones de mujeres o los ecologistas, ha perfilado en el panorama mundial un nuevo aire de protesta y luchas sociales.

La continúa inconformidad alrededor de los poderes que toman las grandes decisiones de tipo económico, como el Banco Mundial, el Fondo Monetario Internacional, la OMC o el G8, ha estimulado la congregación de diversos sectores sociales y, principalmente, ha facilitado que estos diferentes puntos de vista se pongan de acuerdo (cf. Seoane y Taddei, 2001; Houtart, 1999, 2006; Zibechi, 2003).

Por esta razón, y a causa de la globalización y mundialización, es que las resistencias también se han dado a la tarea de elaboración de teorías sobre estos fenómenos, como es el caso de "las convergencias". Los ejemplos más claros de "convergencias" son los distintos foros mundiales, donde han surgido nuevas corrientes del pensamiento y la idea de que "otro mundo es posible". Se pueden destacar los foros sociales mundiales en Porto Alegre, en Venezuela y otros lugares del mundo. Todos estos fenómenos son los que han despertado gran interés en las ciencias sociales sobre la importancia que hay alrededor de las luchas sociales y les han dado fuerza a conceptos como el de la resistencia, la resistencia civil o la desobediencia civil. Por medio de estos conceptos, se ha querido dejar de manifiesto una serie de características y de cualidades en las acciones llevadas por una persona o serie de personas, que se oponen a las diferentes formas de dominación y opresión existentes en el mundo.

La mundialización del capital y de las decisiones económicas afecta a numerosos aspectos de la vida humana. Sabemos bien lo que esto significa en el plano estrictamente económico, pero es preciso subrayar la penetración de la lógica del mercado en ámbitos cada vez más numerosos de la vida humana como la educación, la salud, la seguridad social, la cultura. Cuando la lógica mercantil penetra en estos ámbitos, debilita el carácter de los mismos en tanto derechos humanos progresivamente conquistados a través de luchas sociales, dejándolos así librados a la solvencia de los individuos. Esta lógica conlleva en sí misma la exclusión de los pobres o, en el mejor de los casos, su reducción a un objeto de asistencia. No es por lo tanto llamativo que, medios de comunicación mediante, cada vez más gente reaccione frente a esta situación. (Houtart, 1999, p. 64) 
Frente a todas estas formas de dominación y opresión han nacido distintas maneras de contrarrestar tales poderes, y al mismo tiempo ha llevado a que diferentes autores traten el tema. Una de las obras de mayor relevancia en este aspecto es la de Scott (2000), en la que de manera muy amplia el autor muestra el sinfín de formas con las que las poblaciones se han opuesto a los poderes y a la dominación. Es una obra de gran valor en la medida en que pone como ejemplos la picaresca, los discursos ocultos, el ocultamiento, la clandestinidad, el refunfuño, los cuentos populares o el simbolismo como fuentes de resistencia y oposición. Sin embargo, la resistencia, al estar inmersa en tantas esferas, ha necesitado estar acompañada y analizada desde otras teorías y proposiciones. En este caso, las mayormente aceptadas tanto en la comunidad académica como en la no académica son las de resistencia civil y desobediencia civil.

Sin querer decir que estos conceptos carezcan de validez o de fuerza, y tampoco demeritando su importancia o relevancia, estos conceptos se potencian gracias a la noviolencia. ${ }^{1}$ Sin embargo, muchos teóricos, políticos y activistas dejan de lado este componente al referirse a dichos procesos. Es más, para ejemplificar y demostrar esta afirmación, se puede ver la poca atención que se le presta a la noviolencia y la manera tan indistinta y escueta como se escribe y se habla de ella. Sin temor a equivocarme, puedo señalar que, en muchas ocasiones, escritos y análisis, la noviolencia pasa a un segundo plano, al igual que el análisis desde métodos y estrategias y desde el componente de lo no armado.

Por ello, el propósito de este artículo es mostrar cómo las percepciones de resistencia, resistencia civil y desobediencia civil toman fuerza gracias a la teoría de la noviolencia. El análisis desde la noviolencia se constituye en algo, aunque no nuevo, sí novedoso, en la medida en que permite profundizar e incorporar nuevos elementos de estudio y entendimiento de las resistencias.

Por esta razón, y con fines principalmente metodológicos, este artículo se divide en tres secciones. 1) La conceptualización de cada uno de estos términos - resistencia, resistencia civil y desobediencia civil- y cómo ellos se alimentan de la noviolencia y de la evolución y construcción del concepto. Este primer apartado es una afirmación y el argumento que se sustentará a lo largo del texto. Es necesario que quede claro y se evidencie que la noviolencia es un sustrato de estas dos nociones. 2) Se resaltará el valor de la paz y la importancia que tiene el factor de lo no armado, resaltando la importancia que hay en el análisis de los medios y fines. Con este apartado, se le dará fuerza a la primera afirmación y el porqué de su relevancia. 3) Se hará una propuesta para el manejo de

\footnotetext{
${ }^{1}$ A lo largo de este artículo se hará referencia y uso de la morfosintaxis de noviolencia (todo junto) en la medida que se considera que no se puede pensar en la tradición de lucha satyagraha llevada a cabo por Gandhi sin tener siempre presente tanto los fines como los medios. No se usarán las morfosintaxis de no violencia (separado) o no-violencia (con guión) en la medida en que se consideran reduccionistas a la hora de abordar la temática. La explicación de cada una de estas formas de escritura de la noviolencia se ofrecerá a lo largo de este texto.
} 
estos conceptos desde las posturas de la noviolencia que parte de los tres autores López Martínez (2004, 2006, 2013), Pontara (1991) y Gandhi (2004), donde se hace una clara diferenciación conceptual que puede tenerse en cuenta y en algunos casos adecuarse a los conceptos, como el de resistencia o resistencia civil. Se mostrará cómo estas teorías pueden significar una ventaja o facilidad a la hora de llevar a cabo análisis politológicos e históricos. Este último apartado concretiza la argumentación y propone el uso tanto de una morfosintaxis como de un punto de análisis complejo, donde se le dé cabida a nuevas formas de interpretación de casos o circunstancias concretas y particulares.

\section{La resistencia y la resistencia civil: conceptos que se nutren de la noviolencia}

Desde hechos como los de Manila en Filipinas en 1986, las manifestaciones alrededor de la cumbre de la OMC en Seattle en 1999 y los foros sociales mundiales en Brasil, Venezuela y en diferentes lugares del mundo, es cuando se han popularizado los términos de resistencia, resistencia pasiva, resistencia civil y desobediencia civil.

En el tema de la resistencia, se puede ver que es uno de los conceptos y de las ideas más amplias y genéricas. Si tomamos como punto de partida una fuente de fácil acceso o popularizada, al igual que reconocida internacionalmente, como lo es la Real Academia de la Lengua Española (RAE), que define la palabra resistencia como:

(Proviene del Latín resistentǐa). 1. f. Acción y efecto de resistir o resistirse. 2. f. Capacidad para resistir. 3. f. Conjunto de las personas que, clandestinamente de ordinario, se oponen con violencia a los invasores de un territorio o a una dictadura. 4. f. En el psicoanálisis, oposición del paciente a reconocer sus impulsos o motivaciones inconscientes. 5. f. Electr. Dificultad que opone un circuito al paso de una corriente. 6. f. Electr. Magnitud que mide esta propiedad. Su unidad en el Sistema Internacional es el ohmio.7 f. Electr. Elemento que se intercala en un circuito para dificultar el paso de la corriente o para hacer que esta se transforme en calor. "8. f. Mec. Causa que se opone a la acción de una fuerza.

9. f. Mec. Fuerza que se opone al movimiento de una máquina y ha de ser vencida por la potencia. (http://dle.rae.es/?id=WAPyoek)

Es de resaltar que esta misma definición viene acompañada de un adjetivo que vendría a ser una definición aledaña y es la de "pasiva", donde la resistencia pasiva es: “1. f. Renuencia a hacer o cumplir algo. 2. f. Mec. La que en una máquina dificulta su movimiento y disminuye su efecto útil; p. ej., el rozamiento, los choques, etc.” (http:// dle.rae.es/?id=WAPyoek).

De esta definición podemos extraer tres puntos de discusión. El primero de los aspectos, el concepto de oposición u oponerse es clave. La relación con una fuerza, circuito, 
corriente, que se contrapone, dificulta o interrumpe otra fuerza o corriente es clara. Esta dificultad del mismo modo se repite en las tres primeras acepciones, siendo en la tercera donde quedan más explícitos aspectos sociales, como grupo de personas que se oponen a invasiones o dictaduras. Teniendo presente en todo momento que esta definición es una ejemplificación de las concepciones tan generalizadas del término, el segundo aspecto que se puede ver es que se tiene en cuenta el factor de la violencia como forma de llevar a cabo dicha pugna. Y el tercer y último elemento es que, intentando un poco solventar las distinciones entre formas de resistencia, la define con el adjetivo de resistencia pasiva. ${ }^{2}$

Dejando a un lado el ejemplo de la generalidad con la que se usa el término desde la misma Real Academia Española, y viendo más su evolución e historia, podemos ver que en la Grecia Clásica la resistencia o el valor de oposición o desobediencia también está presente. Se encuentra en obras como Lisístrata, de Aristófanes, o Antígona, de Sófocles. En la Edad Moderna, pocos años después de la muerte de Maquiavelo, las ideas de desobediencia y desacato se ven de manera mucho más clara en la figura de Étienne de La Boétie y su Discurso de la servidumbre voluntaria o el contra uno (1986). Es una obra de vital importancia en la medida en que evidencia que el poder del rey o el monarca está supeditado a la obediencia de los súbditos, es decir que, hasta que la población no sea consciente de que en ellos se fundamenta el poder del rey, no es posible escapar de una servidumbre voluntaria y de que tan solo uno sea capaz de pasar por encima de la voluntad y termine sometiendo a todos. Por esta razón, se considera que La Boétie es uno de los precursores y forjadores del pensamiento y las ideas de la teoría noviolenta (López Martínez, 2006, p. 79).

La Revolución francesa abrió un nuevo escenario y dejó un precedente muy importante para el derrocamiento del Antiguo Régimen. Aun así las reformas de 1814 y principalmente de 1815 bajo el periodo conocido como la Restauración dejaron claro que el sistema monárquico - absolutismo ilustrado ${ }^{3}$ - no iba a desaparecer hasta prácticamente finales del siglo XIX. En este aspecto, es innegable que, aunque muchas de las ideas revolucionarias de 1789 no llegaron a cristalizarse en la medida en que no le supusieron el verdadero fin al Antiguo Régimen, muchos de sus ideólogos, en la memoria de la sociedad contemporánea y actual, siguen siendo un antecedente tanto teórico como histórico.

Pensadores como William Godwin y su texto An enquiry concerning political justice and its influence on general virtue and happiness de 1793, en pleno apogeo de la Revolución francesa, hace uno de los primeros análisis sistemáticos sobre la

\footnotetext{
${ }^{2}$ Dentro de la evolución y los conceptos de la noviolencia se ha discutido mucho sobre los efectos que tiene la confusión sociopolítica de su semántica, donde muchos de sus preceptos fueron asociados a la pasividad (cf. López Martínez, 2006).

${ }^{3}$ El absolutismo ilustrado hace referencia a la última etapa del Antiguo Régimen cuando los monarcas europeos mantienen sus ideas absolutistas y de control del poder en la figura del rey con las ideas propias de la Ilustración. Son estos monarcas los que concretamente van impulsar esta "restauración".
} 
importancia que tienen los métodos de resistencia sin armas. Para Godwin, "si se retira la obediencia, se viene abajo la trama en la que descansa el gobierno injusto" (Randle, 1998, p. 37). Esta obra toma especial relevancia en la medida en que propone la fundación de una sociedad basada en las ideas de la Constitución jacobina de este mismo año, pero partiendo de unas iniciativas pacíficas. ${ }^{4}$

De igual forma, los acontecimientos no solo se limitan a la herencia dejada por la Revolución francesa, sino que del mismo modo para finales del siglo XVIII y principios del siglo XIX con la revolución industrial se da otro tipo de manifestaciones claras de procesos de resistencia en general, tanto con el uso de las armas como sin el uso de ellas. La condiciones de países más industrializados, como Inglaterra y Francia, que estaban experimentando las formas de capitalismo industrial, la urbanización y la conformación de sindicatos, fue donde se dieron más diversidad de expresiones. La implementación de estructuras de producción sustentadas en el uso de la maquinaria impuso unas nuevas formas de trabajo a las que la población no estaba acostumbrada, puesto que venían de modelos más artesanales como eran los gremios que estaban en decadencia y, sobre todo, muchos aspectos de tipo social y cultural que eran propios de la idiosincrasia del Antiguo Régimen que no se había superado del todo. ${ }^{5}$

En contestación a muchos de los procesos industriales y a lo que implicaba el uso cotidiano de la máquina, es que emergen las ideas del ludismo, que abiertamente ven la máquina como uno de los principales problemas en el choque cultural y económico que están viviendo los trabajadores. Por ello, conscientemente, y en busca de la no cooperación, deciden romper y destruir dichas máquinas. Otro ejemplo de este tipo de organizaciones son el cartismo y el carbonismo en Inglaterra. En general, tanto en Europa como en América, existe gran proliferación de sociedades, en muchos casos ocultas, en las que conspirar contra el statu quo es lo primordial.

A causa de todas estas sociedades y de los acontecimientos que significaron las revoluciones de 1830 es que se puede ver la proliferación de las ideas marxistas y las anarquistas, que fueron las que, en gran parte, predominaron en los sectores populares. Estas dos vertientes, alimentadas también de los procesos revolucionarios de la Revolución francesa, fueron decantándose ampliamente por las ideas de la eficacia y eficiencia en lo armado y principalmente con la idea de que la toma del poder debe realizarse bajo la irrupción violenta de las armas, "el tomar el cielo por sorpresa".

\footnotetext{
${ }^{4}$ La doctrina pacifista ya existía con mucha anterioridad; sin embargo, es en este momento de gran algidez y sobre todo con altos contenidos de violencia cuando una iniciativa como la de Godwin (1793) toma importancia. Es uno de los precursores del entendimiento de la lucha de masas con la importancia de los contenidos pacíficos en la era contemporánea (cf. López Martínez, 2006).

${ }^{5}$ Los modelos de capitalismo industrial y las formas del nuevo realpolitik propias del siglo XIX hacían visibles cada vez más las brechas y las problemáticas sociales que implicaban las nuevas formas de producción y explotación.
} 
Partiendo de las ideas de Randle (1998) a causa del estudio de Tilly (2005), se puede ver que, en términos generales, las formas de resistencia de estos momentos eran principalmente pacíficas. Sin embargo, hay dos cuestiones que las llevan a tender al uso de las armas: primero, que parte de las ideas revolucionarias - principalmente de las corrientes marxistas y anarquistas, como se mencionó-, existe una fuerte convicción de que es necesario la toma del poder por las armas, como lo ocurrido en 1789 con la toma de la Bastilla. Esta idea se incrustará tan profundamente en los idearios revolucionarios que los medios no armados pasarán a ser considerados como ineficientes, inoficiosos y, sobre todo, inocuos. Es tan generalizada esta tendencia que los movimientos y las resistencias pacifistas serán vistas y denominadas posturas moderadas o, como fue llamada por muchos pensadores del momento, utópicas (Randle, 1998, pp. 62-63).

La segunda cuestión que influyó en la proliferación de las luchas armadas y del contenido de la violencia en estas esferas fue que dentro de la misma institucionalidad o autoridad se dieron cuenta de que la represión de los Gobiernos en manifestaciones se llevaba a cabo bajo la convicción de que la defensa y respuesta por parte de los manifestantes sería igualmente agresiva o violenta. Así, los mismos Gobiernos eran incitadores de una escalada de violencia en la que finalmente los mayores beneficiarios serían las Fuerzas Armadas y profesionalizadas. De esta manera, los Gobiernos estaban convencidos de que nadie iba a asumir una agresión sin del mismo modo responder con la misma moneda. Esto dejaba detrás del telón la idea de que cualquier manifestación o resistencia sin el uso de las armas estaba abocada al fracaso.

Prácticamente ninguna de las formas de acción colectiva comunes que hemos analizado es intrínsecamente violenta. El grueso de la violencia colectiva surge a partir de corrientes mucho más amplias de acción colectiva esencialmente noviolenta, y es entonces con frecuencia el resultado de una reacción forzada por un segundo grupo - obra a menudo de fuerzas represivas especializadas al servicio de los gobiernos- sobre la reacción colectiva noviolenta del primero. (Randle, 1998, p. 61)

En esa medida, no es de extrañar que, poco a poco, se fuese dando una distinción alrededor del uso de que eran métodos armados y otros no armados. A los procesos que empezaron a desarrollarse por medios diferentes de las armas se les denominó, en primer momento, resistencia pasiva. Para Randle (1998), el término concretamente aparece en 1819 en el libro de Shelley La máscara de la anarquía, y no era de esperar que se le diera un apelativo más fuerte o más reivindicativo. De esta forma, la resistencia pasiva se convierte en uno de los conceptos más generalizados a lo largo de los siglos XIX y XX (Randle, 1998, pp. 49-50).

A causa de la discusión que gira en torno al concepto de resistencia, se puede ver que se han generado muchas acepciones del término, pero estas definiciones han ido 
variando según contextos y circunstancias. Se pueden encontrar definiciones tanto centradas en la Segunda Guerra Mundial, como lo constituyeron el ejemplo de las luchas partisanas, hasta definiciones en relación con las manifestaciones y movilizaciones ya mencionadas, como las de Seattle o Manila.

Por esta razón, López Martínez (2013) ha desarrollado una tipología de la resistencia, basada en unos ejes de reivindicación y de conflicto en los que postula tres tipos: 1) la lucha contra la dominación colonial, 2) la lucha contra los regímenes autoritarios, dictatoriales y totalitarios y 3) la reivindicación de derechos y libertades democráticas y ciudadanas, por la solidaridad internacional, por la ecología y en defensa de la naturaleza y por otro mundo posible. ${ }^{6}$ Estos se pueden explicar con los siguientes aspectos.

\section{La lucha contra la dominación colonial}

Esta resistencia es una reivindicación y un conflicto que nace, con la presencia y el dominio de los imperios, principalmente europeos. En este sentido, el conflicto emerge -más que por la presencia del "otro"- por el vínculo de dominio y opresión que se crea hacia los pueblos autóctonos o indígenas. Para deshacer estos vínculos de dominio, la lucha está acompañada tanto de combates armados como de no armados. De esta manera, para López Martínez (2013), existen varios ejemplos a lo largo de la historia ${ }^{7}$ en los que se evidencia una resistencia no armada frente a la dominación colonial.

\section{La lucha contra los regímenes autoritarios, dictatoriales y totalitarios}

$\mathrm{Al}$ estudiar estas luchas, se puede ver que el paradigma predominante de la violencia en la historiografía ha difuminado y silenciado el papel de la lucha noviolenta. El hecho de que este tipo de sistemas usen como principales métodos la represión y la restricción de las libertades convierte la resistencia armada en legitimadora para dicha la represión, mientras que a la noviolencia la convierte en una alternativa real. En esta medida, los casos de luchas y resistencias sin el recurso de las armas, aunque han sido oscurecidos, han estado presentes y han sido muy importantes y significativos a lo largo de la historia. ${ }^{8}$

\footnotetext{
${ }^{6}$ Para este autor, está claro que en muchos de estos ejemplos no se da una noviolencia específica, en la que se busca un programa creativo con el adversario, sino que en muchas ocasiones se trata de una noviolencia pragmática. Todos estos ejemplos son importantes en la medida en que han presentado cambios significativos en la humanización del conflicto. Las ideas de noviolencia específica y pragmática se tratarán más adelante (López Martínez, 2013, p. 40).

${ }^{7}$ Algunos de los ejemplos que el autor menciona en el artículo son estos: la independencia de las trece colonias: Cuba (1810-1903), Argelia (1830-1950), Egipto (1805-1922), Ghana (1890-1950), Mozambique (1920-1970), Sudáfrica (1899-1919), la India (1900-1947), Polonia (1860-1900), Hungría (1850-1860), Irlanda (1919-1921), Palestina (1920-2012), entre otros (López Martínez, 2013, p. 37).

${ }^{8}$ Los ejemplos que presenta López Martínez son los siguientes: huelga general en Rusia en 1905, resistencia noviolenta en Holanda en 1940, resistencia de maestros en Noruega en 1940, resistencia de Dinamarca, oposición a Hitler de la organización Rosa Blanca, resistencia civil de las mujeres en Italia en 1943-1945, las campañas contra la dictadura en Salvador en 1944, el apartheid en Sudáfrica, la Primavera de Praga en Checoslovaquia en 1968, el colapso de los regímenes soviéticos y la caída del Muro de Berlín en 1989, entre otros (2013, p. 38).
} 
La reivindicación de derechos y libertades democráticas y ciudadanas por la solidaridad internacional, por la ecología y en defensa de la naturaleza por otro mundo posible

Los casos de resistencias que han hecho uso de métodos de acción noviolenta, para la búsqueda y defensa de los derechos humanos, son muy amplios y se pueden evidenciar tanto en los antiguos movimientos sociales del siglo XIX como en los nuevos movimientos que han surgido a lo largo de los siglos XX y XXI. Son muchas las manifestaciones y las formas en las que la noviolencia ha sido una gran protagonista.

Para ejemplificar tanto las luchas contra el totalitarismo como por los derechos y las libertades, se puede hacer referencia a los análisis de Randle (1998), quien desarrolla una de las definiciones de resistencia que más efecto ha tenido en el mundo académico y que da relevancia a la teoría del satyagraha de Gandhi, pero que se centra principalmente en casos directamente relacionados con fines y sueños hacia y desde los conceptos de democracia occidental. Nos referimos en este caso al concepto de resistencia civil, que distingue claramente entre los métodos violentos y los sin el uso de las armas. La idea de lo civil busca implicar la ausencia de cuerpos militares y, sobre todo, resaltar la potencia de la acción colectiva. ${ }^{9}$

Para Randle, la resistencia civil puede ser entendida como

un método de lucha política colectiva basada en la idea básica de que los gobiernos dependen en último término de la colaboración, o por lo menos de la obediencia de la mayoría de la población, y de la lealtad de los militares, la policía y de los servicios de seguridad civil. O sea que está basada en las circunstancias reales del poder político. Funciona a base de movilizar a la población civil para que retire ese consenso, de procurar socavar las fuentes de poder del oponente, y de hacerse con el apoyo de terceras partes. Sus métodos abarcan desde la protesta y la persuasión hasta la no cooperación social, económica y política, y por último hasta la intervención noviolenta. (Randle, 1998, p. 25)

Por ello, cuando hablamos de resistencia civil, se hace hincapié en la negación de la obediencia y, sobre todo, a cuando se retira el consenso que fundamenta el Gobierno. La resistencia civil así percibida implica dos características esenciales: 1) tratarse de una acción colectiva y 2) procurar el no uso de la violencia. Para este autor, la resistencia civil debe situarse dentro del concepto amplio de la acción noviolenta, el cual incluye tanto acciones individuales como colectivas (explicita la importancia de la noviolencia).

\footnotetext{
${ }^{9}$ Al referirse a la acción colectiva del mismo modo, es importante diferenciar resistencia civil del concepto de protesta, el cual claramente se refiere a un momento concreto y temporal, mientras que el término resistencia deja implícita una idea de continuidad (González Piñeros, 2006).
} 
Los objetivos de esta resistencia pueden ser encaminados a proyectos reformistas, como la supresión de leyes, decretos o eliminación de una injusticia concreta, o con objetivos más ambiciosos y revolucionarios, como derribar un Gobierno, una autoridad o el derrocamiento de un sistema político y social.

Para autores como Randle (1998), Arendt (2005a, 2005b), Habermas (2000) o, en Colombia, Hernández (2004), Ballesteros (2005) y González Piñeros (2006), todos concuerdan en que la resistencia civil se encuentra en un marco más amplio, como los métodos de acción noviolenta. Si se toman algunos elementos de los que se tienen en cuenta para definir la resistencia civil, podemos ver que se relacionan estrechamente con los preceptos y las ideas impulsados desde la teoría de la noviolencia.

Si analizamos los postulados que expone Randle (1998), y muchos de los autores ya mencionados, se puede ver que existen grandes avances en el desarrollo de sus definiciones; sin embargo, del mismo modo, se puede observar que muchos de estos elementos son anteriores, y se sedimentan gracias a las ideas desarrolladas por David Thoreau. Generalmente, se hace referencia a Thoreau por acuñar el concepto de desobediencia civil; sin embargo, en el momento de desarrollar sus ideas, y presentar su primer ensayo en 1848 y su posterior publicación en 1849, no se titulaba como lo conocemos hoy en día Sobre el deber de la desobediencia civil, sino que se titulaba Resistencia al gobierno civil. Es solo hasta 1866, cuatro años después de su muerte, que se le da el título definitivo que conocemos ahora (Herranz, 1992, p. 21). De esta manera, aunque el fenómeno de desobedecer o de resistir frente al Gobierno es más algo antiguo, son los postulados de Thoreau los que más han influenciado en las teorías y los pensadores de habla inglesa, porque, además de referirse y partir de un caso como el norteamericano en el que se puede hablar de una resistencia contra un gobierno "civil", también tiene en cuenta una visión pragmática y moral de la resistencia, por lo que ha sido estudiado por pensadores como Sharp, que se centran en lo pragmático, hasta personalidades como Gandhi, que, sin perder de vista los métodos, les da mayor importancia a los valores.

Bajo la misma tradición de "lo civil" y el pensamiento de Thoreau se fundamentan los conceptos de resistencia civil y desobediencia civil, dado que parten de la misma naturaleza: desobedecer a la injusticia proveniente de un Gobierno. Si se entra en detalle, el concepto de desobediencia civil tiene una connotación específica y es la de encontrarse en contraposición con una norma o una ley establecida, que se considera injusta o nociva (Ballesteros, 2005). Se diferencia del concepto de resistencia civil en la medida en que puede ser entendido como un método dentro de ella, es decir, la desobediencia civil puede ser una forma de acción dentro de la misma resistencia civil. La desobediencia civil, al igual que la resistencia civil, hacen parte de la estructura más amplia, como es la del pensamiento noviolento (reafirmación de la argumentación y novedad del texto). 
El concepto de resistencia se trasformará y empezará a tomar gran valor y relevancia a partir de Gandhi, quien reivindica su condición activa y valiente y genera toda una teoría y un entramado que denominó satyagraha.

\section{El valor de lo no armado: el análisis de los métodos-medios y las estrategias-fines}

En este apartado en ningún momento se trata de demeritar el uso de otras herramientas de análisis o desprestigiar otros valores. Simplemente, es un esfuerzo por darles potencia a factores como el no uso de las armas como uno de los grandes determinantes de las luchas sociales y políticas en la actualidad, al igual que a la relación entre los métodos y las estrategias (acciones y fines). La penetración y permeabilidad de la violencia en la sociedad, y más en una sociedad como la colombiana, hace pensar con mayor optimismo que el desmonte de la violencia en las estructuras sociales es fundamental. Por esa razón, pensar, estudiar y proponer en claves de paz es un camino coherente y necesario. Si partimos de la importancia del contenido no armado y la relevancia de los medios-fines, podemos referirnos principalmente a cuatro ideas clave:

1. La violencia históricamente ha sido uno de los paradigmas que ha primado. La violencia en el imaginario colectivo se ha venido representando como lo activo y eficaz. Se ha concebido como la única forma y medio por el que se desarrolla la política y las relaciones sociales. Esto ha imposibilitado que valores como la paz sean introducidos en las agendas internacionales como un aspecto de vital importancia. En esa medida, cambiar los valores o las perspectivas para la paz se convierte en asunto prioritario para diferentes corrientes del pensamiento ${ }^{10}$ que ven en este valor un punto de partida significativo en la búsqueda y transformación de programas constructivos y de acción.

2. Las experiencias y las acciones emprendidas desde lo no armado, en términos generales, han influido en las formas de ver las luchas sociales y se han distinguido de manera clara y evidente. Aunque existan líneas muy delgadas en la decisión del uso o no de las armas, su uso o no sí ha marcado una diferencia. Un ejemplo de esto lo podemos encontrar ya desde los tiempos de Gandhi, cuando las autoridades coloniales no sabían cómo denominar la lucha, pero aun así sabían concretamente de que no se trataba de revueltas y huelgas convencionales. También conocían que el hecho de que la fuerza de estos movimientos no se soportara en la supremacía militar y armamentística dificultaba y desconcertaba a la Corona y a las autoridades británicas.

\footnotetext{
${ }^{10}$ Es importante en este aspecto tener en cuenta los estudios para la paz o peace research. Se pueden encontrar algunos de estos presupuestos de la epistemología de los estudios para la paz en Galtung (2003).
} 
De esa manera, la primera forma que ingeniaron los ingleses para denominar estas luchas fue la de "resistencia pasiva", que se había usado ya con anterioridad en el siglo XIX, como lo evidencia Randle (1998). El mismo Gandhi y sus seguidores se esforzaron en mostrar las diferencias entre una lucha pasiva y la lucha que llevaban a cabo. Costó mucho esfuerzo crear un concepto como el de satyagraha y, sobre todo, poder mostrar que no se trataba de actos de pasividad. La misma Corona desde el primer momento intentó por todos los medios desprestigiar y desvirtuar la lucha de Gandhi, y una de las formas que encontró fue con denominaciones como la de la pasividad, que no expresaba fidedignamente lo que realmente determinaba. En palabas de Gandhi, "la no colaboración no es una disposición pasiva, sino un estado intensamente activo, más activo que la resistencia física o la violencia. La denominación 'resistencia pasiva' es errónea" (2004, p. 268).

Para Gandhi, el no-matar, o causar daño o sufrimiento (ahimsa), es imprescindible, puesto que para él "no hay que destruir a los hombres sino a los métodos que estos hombres llevan a cabo como la dominación, la exclusión o la guerra” (1998, p. 58).

De esta manera, la relación entre los medios y los fines toma protagonismo para las ideas de Gandhi. El uso de medios y métodos de imposición o violencia corrompe y desvía los fines. Los medios con los que se decidan hacer las acciones son el único garante de que los fines sean loables. Y del mismo modo son lo único palpable y tangible, puesto que los fines y las estrategias es donde se quiere llegar y, por ello, por más que se pretenda magnificar dicho fin o estrategia, no estará presente sino hasta el final.

Tomando como punto de partida y fuente el artículo "Gandhi, política y satyagraha" (López Martínez, 2012b), podemos extraer, a grandes rasgos, lo que significa el método de la lucha satyagraha y los elementos que hacen parte del complejo pensamiento gandhiano. Podemos entender la lucha gandhiana a partir de seis principios:

1. Satya (verdad): es entendida desde dos perspectivas:

a. Verdad epistemológica, siendo esta consciente de que en determinado momento puede cambiar dicha verdad.

a. Una verdad ontológica, entendida como algo mucho más profundo, el ser, lo absoluto, el amor, la guía, Dios. Para él, se asocia el concepto de Dios a verdad, a la suma de las cosas que viven. Por esta razón, el concepto de satyagraha es la búsqueda de la verdad, del paso de la búsqueda de una verdad epistemológica a una ontológica.

2. Ahimsa: deriva del sanscrito de la raíz hims, entendida como "dañar, herir, matar", sino que a esta raíz se le ha añadido el prefijo que implica su negación, ahimsa vista como total ausencia o deseo de dañar, odiar, hacer el mal, matar a cualquier ser viviente (inocencia o pureza). 
3. Sarvodaya: se puede interpretar como el bienestar para todos, y no solo para la mayoría. En Gandhi, lo podemos ver como su "programa constructivo", el gran "pacto social” que él quería para la India, fundado en unas tradiciones y unas raíces culturales.

4. Swaraj: derecho y acción de la autoderminación, autonomía, independencia política. Para López Martínez,

el objetivo no es solo la liberación de la India de los ingleses, sino la liberación de todas las naciones y los individuos oprimidos y explotados dondequiera que estuvieran. Ese es el verdadero y profundo sentido del swaraj: "es el gobierno de todo el pueblo, es el gobierno de la justicia”. (2012a, p. 51)

5. Swadeshi (pertenencia al propio país). Hace referencia a la autosuficiencia o autarquía económica y a los modos de hacer locales y artesanales. Tiene implicaciones tanto económicas como culturales, es valorar lo propio sin menospreciar lo ajeno.

6. Satyagraha: es la búsqueda, persistencia, la fuerza por la verdad.

Estos seis principios son los que en gran medida configuran la magnitud de la lucha. Por esta razón, se puede hablar de que empieza a haber una verdadera transformación en las maneras de ver y entender la política, y no solo en una manera de responder pasivamente ante los conflictos. Non-violence deja definitivamente expuesta la importancia del no uso de las armas. En este caso, los medios pasan a tener una relevancia vital:

Del mismo modo que la violencia es solo un medio; el fin es la verdad. Para que los medios sigan siendo medios tienen que estar siempre a nuestro alcance. Por eso la noviolencia es nuestro deber supremo. Si cuidamos los medios, antes o después alcanzaremos el fin. Una vez comprendido este punto, la victoria final es incuestionable. (Gandhi, 2004, p. 46)

El caso del gandhismo en la India, al constituirse en una experiencia ejemplar, empezó a ser tenida en cuenta por activistas, filósofos y académicos. En primer lugar, el pensamiento gandhiano se constituye como una doctrina filosófica y de acción, en la que por sus resultados estratégicos y su alternatividad da pie a la generación de estudios académicos, de los que se han nutrido posteriores teorías, como los estudios poscoloniales, alrededor de los "subalternos", ${ }^{11}$ o los estudios para la paz o peace research. En

\footnotetext{
${ }^{11}$ Los ejemplos más representativos los encontramos tanto en las corrientes posestructuralistas como en teorías poscoloniales. Dentro de los estudios poscoloniales es importante destacar la labor de los estudios subalternos y su papel a la hora de intentar darles voz a los silenciados por la historiografía colonial. Alrededor de los subalternos se han generado toda una teoría y una discusión. Aunque el concepto de subalternos fue utilizado en
} 
segundo lugar, el pensamiento de Gandhi no es una teoría académica, sino una aportación epistemológica, que cuestiona los modelos de la historia, la sociología y la ciencia política, instruidas bajo la hegemonía de la violencia. En tercer lugar, es importante aclarar que existen varias perspectivas para tratar el tema de las luchas sociales. Una de estas perspectivas es mediante el estudio de los movimientos sociales y los nuevos movimientos sociales. En esta vertiente, podemos destacar la escuela estadounidense, que parte de los presupuestos de la elección racional, donde destacan autores como Tilly (2005). Y la escuela europea, donde se centran en cualidades como la identidad, la participación política, la autodeterminación, los derechos, entre otros, y destacan autores como Touraine (1995), Arendt (2005a, 2005b), Habermas (2001), entre otros. Se podría, de igual modo, hablar de una tercera vertiente que no niega la incompatibilidad de estos dos puntos de vista a la hora de estudiar los movimientos o nuevos movimientos sociales (González Pañeros, 2006).

De la escuela estadounidense se puede resaltar que los parámetros de interpretación en los que se centran son a partir del estudio de estrategias y motivaciones de la acción. Podemos resaltar cinco parámetros de análisis: 1) la movilización de recursos, 2) la estructura de oportunidades políticas, 3) la contextualización externa, 4) las rupturas estructurales y 5) la identidad colectiva (González Pañeros, 2006).

Estos análisis son de vital importancia en la discusión de las luchas sociales. Les ha permitido a las ciencias sociales incluir variables de diversas características para el análisis, y entre esa pluralidad, el factor de lo no armado también ha estado presente. Aunque no se ha analizado desde la misma perspectiva de la que podría hacer Gandhi, sí se ha tenido en cuenta como variable de carácter estratégico. ${ }^{12}$

Sin embargo, la perspectiva primordial desde la que se parte en este artículo es la de los estudios para la paz. Son buen ejemplo del trabajo y la discusión que se empezó a generar desde la academia sobre los temas de la paz la Journal of Conflict Resolution, en 1957, o el Centro para la Investigación y la Resolución de los Conflictos, en 1959, en

primera instancia por Gramsci (1934) al referirse a los grupos de población que estaban supeditados a un poder hegemónico determinante, es en realidad con los aportes de Guha (2002), Bhabha (1994), Fanon (2009), Said (1990) o Spivak (1997) cuando este concepto se transforma en un punto de partida para el análisis —objeto de estudio- y se desliga de la tradición revolucionaria de finales del siglo XIX y principios del siglo XX en Occidente. Dentro de la misma teoría de la subalternidad existen detractores que, aun partiendo de estas ideas y bases, consideran que el concepto de subalterno es condicionante y coercitivo. Spivak, en ¿Pueden hablar los subalternos?, hace una fuerte crítica, mostrando que la condición de subalternidad se determina en muchos sentidos por la voz acallada de ellos. Sin embargo, cuando estos tienen voz o la consiguen, rompen con su condición de subalternidad. Esta y otras ideas se conectan mucho más con las concepciones de autodeterminación y poder de las que parte Gandhi. En definitiva, se puede decir que, aunque no vayamos a partir de estos postulados, es bastante interesante tenerlos en cuenta en la medida en que se han constituido en todo un boom y, por ende, en un precursor e impulsor del conocimiento y la crítica científica.

12 Un ejemplo de que sí se le ha dado importancia al tema no armado lo encontramos en Tilly (2005) y Arendt (2005a, 2005b). 
los Estados Unidos (Harto de Vera, 2004), o en Suecia y Noruega, en 1964, el Instituto de Investigación sobre la Paz de Oslo y la revista Journal of Peace Research (Fisas, 2004). La figura de Johan Galtung en este caso será la más relevante y reconocida en el ámbito académico e investigativo. ${ }^{13}$

En esa búsqueda de incidir de manera directa en el panorama político, social y económico, la investigación para la paz también se ha encargado de dejar claras sus bases epistemológicas, en la que Galtung de manera clara afirma:

La investigación empírica sobre la paz nos informará sobre las pautas y condiciones para paz/violencia en el pasado, dado que solo el pasado puede aportar esos datos. Los cánones de la investigación son los mismos que para otras ciencias sociales: recolección cuidadosa de datos, procesamiento, análisis y formación inductiva de teorías; o, dándole la vuelta, deductivamente, comparación de datos y teorías, ajustando las segundas a las primeras para obtener consonancia entre unas y otras. (2003, p. 32)

Gracias a estas investigaciones se configurará la nueva corriente de investigación para la paz, en la que ya no solo se tienen en cuenta los aspectos técnicos y prácticos, sino también las estructuras y las relaciones necesarias para la consecución de una paz que sea no solo negativa. Las nuevas realidades exigían nuevas estrategias de construcción de paz; los nuevos enfoques tendrán que tener en cuenta las dimensiones sociales, culturales, políticas y económicas, que permitan la prevalencia de una paz positiva a “escala popular" en vez de darles protagonismo o reservar el derecho de solucionar los conflictos a los Gobiernos (Ledereach, 2000).

La importancia del giro epistemológico es que la violencia pase a un segundo plano y no sea la forma de medir el éxito de las luchas sociales. Se hace imperativo darles un papel protagónico a unos valores como el de la paz, con unos principios más constructivos y propositivos. La convicción de que solo cuidando los medios (puesto que son los únicos palpables) es la que ratifica la idea de que realmente se pueden alcanzar dichos fines.

Algunos dicen: "Después de todo, los medios no son más que medios". Yo prefiero decir:

"A fin de cuentas, todo depende de los medios". Los medios determinan el fin. No hay ningún muro de separación entre los medios y el fin. De hecho el creador nos ha dado el

\footnotetext{
${ }^{13}$ En el seno tanto de esta revista como de este instituto, se fundamentarán muchos de los pilares de tales teorías, por ejemplo la redimensión del concepto de violencia como es concebir sus diferentes facetas, como la violencia directa, estructural o cultural, al igual que las dimensiones de la paz, ya sea positiva, ya sea negativa. De estas investigaciones también se fundamentará la teoría en las intervenciones internacionales donde se hablará de conceptos como el peacekeeping en cuanto operaciones armadas para el mantenimiento o la vuelta de la paz, el peacemaking en cuanto la negación entre los contendientes para solucionar el conflicto o peacebulding en cuanto la reconstrucción política de la paz (López Martínez, 2013).
} 
control (y muy limitado) sobre los medios, no sobre el fin. La consecución de la meta es exactamente proporcional al buen uso de los medios. Y esta es una regla sin excepción. (Gandhi, 2004, p. 72)

Cuarto, si se analizan las primeras ideas provenientes del gandhismo y su transformación en el concepto de noviolencia, podemos ver que existen varios autores que han aportado a la construcción del término, entre ellos: Gene Sharp, Clarence Marsh Case, Jean-Marie Muller, Aldo Capitini, Giuliano Pontara, Mario López Martínez. Aunque aparentemente esta temática dentro del campo de las ciencias sociales ha sido poco tratada, en términos generales hay políticos, activistas e ideólogos que han tratado y hablado sobre la noviolencia. Esta constante discusión y construcción ha estado del mismo modo condicionada por los diferentes momentos históricos y culturales.

En las décadas de 1920 y 1930, muchos políticos y activistas ya eran conocedores de las experiencias llevadas a cabo por Gandhi (Clarence Mars Case, Richard Gregg, Aldo Capitini, etc.). Por esta razón, en la Segunda Guerra Mundial, ya se ven ejemplos claros de resistencias sin el uso de las armas, aunque evidentemente dentro de la historiografía se resalta mayoritariamente las resistencias armadas.

Si vemos en este sentido los aportes realizados en relación con las resistencias frente a los regímenes autoritarios y totalitarios, algunos académicos, como Sémelin (1989), analizando la Segunda Guerra Mundial, en Unarmed against Hitler: Civilian resistance in Europe, 1939-1943, dan un importante paso en llenar vacíos de la historia. En muchos casos, la resistencia civil se entendió como un "acompañamiento" a unas resistencias armadas. En ese sentido, nuevamente se pierden contenidos tan importantes como el que desempeñó la población por medio de huelgas o boicots. El "acompañamiento" difumina factores como la "legitimidad" o la "cohesión social".

Para López Martínez (2012a), dentro del análisis de estas luchas contra los totalitarismos, la experiencia italiana representa un gran ejemplo de la importancia de lo no armado. En medio de un conflicto tan de alta intensidad como lo fue la Segunda Guerra Mundial, emergieron figuras como la de Aldo Capitini, gran teórico convencido de la noviolencia y detractor activo del fascismo de Mussolini. También destaca el desarrollo teórico alrededor de la lucha no armada a través de lo que constituyeron las luchas partisanas y el papel de cuerpos civiles no solo como colaboradores, donde pone como ejemplo a teóricos como Lidia Menapace o Claudio Pavone quienes hablan del tema. En este desarrollo teórico de las luchas no armadas, es importante destacar el papel de lo que López Martínez (2012a) denomina las "mujeres en la resistencia civil", donde Anna Bravo y Anna María Bruzzone (1995) hacen dos innovaciones: la inclusión de las mujeres en la historia y el papel de ellas como predominancia de lo no armado. Estas autoras matizan y hacen referencia a la "resistencia civil no armada". 
El trabajo de académicos centrados en las luchas por los derechos y las libertades se puede evidenciar nuevamente en los estudios de Randle y su "resistencia civil", la cual deja claro que está enraizada en los presupuestos de Gandhi y la satyagraha. De esta manera, se puede ver la importancia que dentro de la teoría noviolenta tiene el factor de lo no armado y, sobre todo, cómo en la actualidad incluir dicho factor ha dado grandes frutos y nuevas perspectivas de análisis.

A manera de síntesis, se puede decir que es importante y relevante la idea de la resistencia no armada y la congruencia que debe existir entre los medios y los fines. La resistencia con el adjetivo civil a veces no deja claros aspectos como la dejación de las armas y en esa medida ha sido necesaria la inclusión de otros adjetivos o de otros constructos, como el de la noviolencia.

\section{Conceptualización de la noviolencia: propuesta para la complementariedad}

Parte de las problemáticas que han motivado estas líneas es ver cómo en diferentes publicaciones académicas, en artículos periodísticos, en artículos de opinión y en diferentes fuentes se usa de manera indistinta la escritura de la noviolencia, sin mencionar la que se le da a la de resistencia.

Algunos académicos se han preocupado por distinguir, y conceptualizar alrededor de la noviolencia, y a partir de esa discusión es recomendable seguir teniendo en cuenta estos elementos en conceptos como el de resistencia o resistencia civil. Como se mencionó en el apartado anterior, buscar darles fuerza y relevancia a valores como lo no armado o lo noviolento puede ser un buen principio.

Partiendo de los postulados de López Martínez, de Giulliano Pontara y principalmente de Gandhi, se puede evidenciar que existen diferentes formas de entender y escribir la noviolencia. Entrando en el nivel más didáctico y comprensivo, como el de López Martínez, que nos habla de la morfosintaxis del concepto, podemos ver que existen tres formas de escribir la noviolencia. La primera y más común es "no violencia”, separado, donde esta escritura solo deja implícita la ausencia de violencia; es una simple negación. La segunda forma es no-violencia, con guion; en este caso, ya no se entiende como la simple negación, sino que se le atribuye un conjunto de métodos o medios de acción individual o colectiva, sin el uso de armas. ${ }^{14}$ Los principales representantes de esta forma de entender la no-violencia como la sistematización, la catalogación y el análisis de su eficiencia y eficacia son Gene Sharp y Clarence Case de la Universidad Estatal de Ohio y la Universidad de Iowa, respectivamente. La tercera forma de referirse a la noviolencia es uniendo las dos palabras, donde no solo se centra en el conjunto de unas formas de

${ }^{14}$ Esta acepción es la más tradicional y sus antecedentes pueden encontrarse en el concepto de pax romana donde existe simplemente una contraposición o ausencia de la violencia. 
acción o actuación, sino que parte de la idea de que puede llegar a constituir todo un “programa constructivo” (López Martínez, 2006, 2013; Pontara, 1991) (tabla 1).

\section{Tabla 1. Tipologías de la noviolencia según autores}

\begin{tabular}{|l|l|l|l|}
\hline \multicolumn{1}{|c|}{ Autor } & \multicolumn{3}{c|}{ Tipologías } \\
\hline Mario López Martínez & Noviolencia & Noviolencia & Noviolencia \\
\hline Guilliano Pontara & Noviolencia pragmática o negativa & Noviolencia específica o positiva \\
\hline Gandhi & $\begin{array}{l}\text { Noviolencia del } \\
\text { cobarde }\end{array}$ & $\begin{array}{l}\text { Noviolencia } \\
\text { del débil }\end{array}$ & Noviolencia del fuerte \\
\hline
\end{tabular}

Fuente: elaboración propia

El segundo autor del que podemos hablar es de Giuliano Pontara. Para él, existen dos formas o acepciones de tratar el concepto de la noviolencia. La primera acepción debe ser entendida exclusivamente como un "modo de actuar" o un conjunto de métodos y técnicas, y la segunda se refiere a un "conjunto de ideas, conceptos, teorías y propuestas de estrategia política" (1991, p. 1054).

En este sentido, la primera acepción la llama noviolencia pragmática o negativa, ${ }^{15}$ en la que

nos referimos a un conjunto de métodos de lucha, b) caracterizados negativamente como exentos de violencia y c) cuyo empleo es de por sí compatible con cualquier doctrina o ideología por cuanto nada excluye que puedan ser empleados, por razones tácticas, por cualquier grupo con vistas a cualquier fin o por cualquier causa, sea justa o injusta. (p. 1054)

Frente a esta primera acepción podemos encontrar gran variedad de ejemplos ilustrativos, pues durante la historia han existido numerosos casos en los que se han utilizado los métodos y las acciones políticas noviolentas sin existir compatibilidad con sus fines. No obstante, podemos destacar dos ejemplos históricos claros. El primero es el caso del nazismo en sus primeras etapas, en las que se utilizaron acciones propias de la noviolencia de manera pragmática, para evitar una confrontación con los cuerpos de seguridad del Estado del momento, tras lo cual se produjo una enorme incompatibilidad con los fines de la noviolencia, como fueron, no solo los genocidios, sino todos los efectos devastadores de la guerra. El segundo ejemplo podemos encontrarlo, en un ámbito más local y actual, como sucedió en Bogotá, en las huelgas de hambre y las sentadas que llevaron a cabo en 2014 diferentes activistas para que se volviesen a permitir las

\footnotetext{
${ }^{15}$ Como mencionamos, a este concepto López Martínez le da el término de genérico, el cual expresa la misma idea y hace referencia al uso compatible con cualquier ideología, por lo que a efectos prácticos se usaran indistintamente los dos términos.
} 
corridas de toros en la plaza de toros de la Santa María. Es evidente que el asesinato de un animal en medio de una plaza resulta incongruente con la noviolencia.

En este orden de ideas, como segunda acepción, vemos la noviolencia específica o positiva, la cual se entiende como

un ordenamiento político fuertemente descentralizado, cuyas estructuras básicas están constituidas por consejos de ciudadanos organizados por fábrica, barrio, pueblo, etc., provistos de amplios poderes decisionales, fundados en el método democrático por lo que respecta a las cuestiones de política local, provistos de un poder efectivo sobre las decisiones que consideran a toda la comunidad. La doctrina noviolenta teoriza ampliamente la socialización de los grandes medios de producción, para los que auspicia la autogestión por parte de los trabajadores, pero deja en cambio abierta la cuestión acerca del grado en que ha de estar planificada la economía en una sociedad tal. (Pontara, 1991, p 1057)

Frente a esta acepción el autor afirma que pertenece a una definición determinada de una concepción ético-política específica, es decir que guarda unas particularidades únicas y propias de un entramado noviolento, como el que en gran parte propugnan Gandhi y sus adeptos al referirse al concepto de satyagraha.

Dentro de esta misma acepción, Pontara también muestra que esta noviolencia propugna

una sociedad con bajo consumo de energía y en la cual el desarrollo tecnológico esté del todo considerado por las exigencias de un pleno y armonioso desarrollo de toda la persona [y por] la abolición de todo tipo de ejército y confiar la defensa de la sociedad que propugna la resistencia noviolenta de masas. (p. 1057)

Asimismo, considera que, basándose en el principio weberiano de la ética de la responsabilidad, en la noviolencia, se parte del principio del no uso de la violencia, sobre todo de la organizada, como instrumento de lucha política, porque a partir de esto se condena la llegada de consecuencias negativas y desencadenadas, como:

- El proceso de escalada histórica de la violencia.

- Las tendencias deshumanizadoras y brutalizantes anexas a la violencia.

- La pauperización del fin que puede conducir el empleo de ella.

- La violencia organizada favorece el surgimiento y asentamiento en lugares cada vez más importantes en la sociedad o del movimiento de individuos y grupos autoritarios, caracterizados por un impulso inmediato al uso de la violencia y partidarios de la militarización del movimiento y de la sociedad en la que han surgido. 
- Se evidencia un proceso de instituciones cerradas, jerárquicas y autoritarias, por lo que la violencia tiende a convertirse en un componente estable e integral del movimiento o de la sociedad en la que incurre (Pontara, 1991).

Finalmente, el último autor al que nos podemos referir es precisamente quien consolida y simplifica todos estos pensamientos: Gandhi. Él distingue tres tipos o formas de noviolencia. Primero, la noviolencia del cobarde, que puede ser asociada como tal con la pasividad, que ocurre cuando no hay confrontación, se huye, no se espera y se resiste, sino que se renuncia a luchar. Segundo, la noviolencia del débil, que es aquella que recurre a los métodos de lucha no armada por hechos o razones circunstanciales, y son el preámbulo o la espera del uso de los recursos armados y la toma del poder. Y tercero, la noviolencia del fuerte o satyagraha (López Martínez, 2006, p. 154). Refiriéndose a la noviolencia del cobarde, del débil y de los fuertes, afirma:

Los hombres de un poblado cercano a Bettiah me contaron que habían huido mientras la policía saqueaba sus casas y acosaba a sus mujeres. Cuando me dijeron que habían huido porque yo les había dicho que fueran noviolentos, bajé la cabeza avergonzado y les aseguré que no era ese el significado que yo daba a la noviolencia. Y les expliqué que lo que esperaba de ellos era que se interpusieran para recibir los golpes más fuertes dirigidos contra quienes estaban bajo su protección, que hicieran recaer sobre sus cabezas todo el daño, sin deseo de venganza y dispuestos incluso a morir, y que nunca huyeran del centro del huracán. Es noble defender los bienes, el honor y la religión a punta de espada; es más noble aún defenderlos sin tratar de hacer daño al malhechor; pero es vil, antinatural y deshonroso abandonar el lugar donde estar y, para salvar la piel, dejar los bienes, el honor y la religión a merced del malhechor. A quienes saben morir sé cómo enseñarles el mensaje de la noviolencia, pero no cómo enseñárselo a quienes tienen miedo a la muerte. (Gandhi, 2004, p. 208)

La misteriosa eficacia de la noviolencia no se mide por sus efectos visibles. Pero no nos conformamos mientras se permita que el veneno del odio impregne la sociedad. Esta lucha es un maravilloso esfuerzo de conversión. Nuestro objetivo es nada menos que la conversión de los ingleses. Y esta nunca se logrará si abrigamos con mala voluntad a la vez que aparentamos seguir el camino de la noviolencia. Así pues, quienes quieren seguir la senda de la noviolencia y, sin embargo, tienen mala voluntad, tendrán que volver sobre sus pasos y arrepentirse del mal que se han hecho a sí mismos y que han causado al país. (p. 207)

Por otra parte, frente a la noviolencia como virtud de los fuertes, del mismo modo señala: 
La noviolencia y la cobardía son incompatibles. Puedo imaginarme a una persona que, a pesar de estar armada hasta los dientes, sea cobarde en su corazón. La posesión de armas supone un cierto miedo y hasta una cierta cobardía. La verdadera noviolencia es imposible para quien no es auténticamente intrépido. (p. 207)

Habiendo aclarado las diversas tipologías y acepciones, se puede ver que, a lo largo del presente texto, se ha optado por referirse a la noviolencia (todo junto) en la medida en que la concibe como un todo que no puede desligarse entre métodos y fines. También es necesario aclarar que, cuando se hace referencia a simples métodos o su estudio, se pone por separado o con guion. Como este estudio se ha basado principalmente en los planteamientos y las formulaciones llevadas a cabo por Gandhi, es ineludible y preciso hablar de noviolencia.

La importancia de esta morfosintaxis o de distinguir estos tipos de noviolencia está en que permite identificar tanto otras problemáticas como sus posibles soluciones. En ese sentido, las ventajas que tiene incorporar estas categorías son principalmente:

1. Dar más valor y peso a los actores de la sociedad civil o a los movimientos sociales frente a los actores armados legales e ilegales, no solo a la ciudadanía en general, sino a grupos específicos, como mujeres, afrodescendientes, indígenas, entre otros colectivos.

2. Analizar el posible final del conflicto desde la perspectiva de que llegará, no solo por un acuerdo entre los "señores de la guerra", sino por un cambio profundo y cultural del papel poco útil de la violencia a la hora de plantear procesos de modernización y cambio.

3. Hacer énfasis en el estudio y análisis de los comportamientos marginales o minoritarios, tales como las tipología de acción noviolentas, múltiples formas de resistencia y desobediencia, acciones simbólicas y creativas en defensa de la vida en medio de la guerra, etc., constatando que tales referencias, aunque fueron minoritarias en un principio, se han ido extendiendo a más y más grupos sociales de la ciudadanía.

4. Para partir de premisas epistemológicas y axiológicas en las que el concepto de paz se amplía de manera semántica — su significado- y adquiere un valor social más comprometido y exigente, de ahí que la paz no se identifique solo - para esta literatura- con el final del conflicto armado interno o con procesos exitosos de justicia transicional, sino con cambios estructurales y culturales profundos, entre los que no solo están los cambios institucionales - política, justicia, administración-, sino también cambios culturales, interculturales y de género.

Además de estas ventajas fundamentales, esta morfosintaxis también sirve para: 
1. Identificar la importante relación entre métodos y estrategias.

2. Considerar el grado de autonomía de la resistencia (si está manipulada o no) y si busca una relación con otras estructuras, como las estatales, o si, de lo contrario, busca su independencia y autodeterminación.

3. Examinar el grado de disciplina.

4. Estudiar el grado de organización.

5. Tener en cuenta su continuidad.

Para finalizar, es pertinente hacer referencia a la aplicabilidad de estas teorías a hechos y casos particulares. En el caso concreto de este artículo, se puede decir que existen unas motivaciones y preocupaciones personales que han permitido indagar sobre estos aspectos. Muchas de estas ideas son producto de la tesis doctoral Caminando hacia la satyagraha: comunidad, lucha y kweet fxindxii en los nasas (1972-2015) (Martínez Bernal, 2016), que versa sobre la resistencia y la comunidad nasa en el norte del departamento del Cauca (Colombia), desde una óptica de los estudios para la paz y la noviolencia.

En esta investigación, se parte de las nociones e ideas de la noviolencia como fuente de análisis, para ver sus similitudes y divergencias, la aplicabilidad de la teoría a un hecho concreto y real, la relación y congruencia entre medios y fines, la importancia de los programas constructivos - para el caso de los nasas, planes de vida-y las fortalezas y debilidades que presenta dicha resistencia.

La noviolencia, en este sentido, sirve como fundamento para un estudio mucho más amplio de lo que significan estas resistencias, y cómo estas iniciativas se configuran como alternativa noviolenta ante la guerra en Colombia. La noviolencia permite ver la importancia que le prestan las poblaciones indígenas a lo no armado (aimsha), a lo que constituye el bienestar o armonía de los pueblos y la construcción de sus planes de vida (sarvodaya), a lo que implica la autonomía y la autodeterminación (sawraj), a lo que constituye la memoria y las tradiciones culturales (swadeshi) y a lo que significa la pervivencia y convivencia con los demás seres basados en unos planes de vida (satyagraha).

Aunque existen otras teorías y perspectivas para tratar estos hechos, ${ }_{16}^{16}$ la noviolencia es un sustrato amplio, innovador y preciso. Permite generar categorías de análisis, encontrar relaciones y divergencias, combinar conceptos y, sobre todo, dar concreción a los resultados. De esa forma, se puede determinar si dichos hechos de resistencia civil son no violentos, no-violentos o noviolentos. La inclusión de esta morfosintaxis permite

\footnotetext{
${ }^{16}$ Dentro de los mismos estudios para la paz existen diversas formas de tratar estos conceptos. Un ejemplo de ello lo constituyen las teorías de la paz imperfecta desarrolladas por Muñoz (2001). Aunque esta teoría puede considerarse de gran relevancia, en muchas ocasiones puede pecar de exceso de amplitud, en la medida en que cualquier iniciativa en pro de la paz puede estar dentro de dicha teoría, y en ese sentido cabrían dentro de la esta demasiados aspectos.
} 
ver si se trata de un proceso en el que simplemente hay ausencia de violencia, si se trata de un uso de medios con intenciones meramente tácticas (no-violencia) o si se trata de un conjunto de métodos y fines que representan un todo. O en las palabras de Pontara, se pueden determinar unas particularidades únicas y propias de dicho entramado.

Centrarnos en las comunidades indígenas del norte del Cauca, nos ha permitido indagar sobre la naturaleza de su resistencia. Por esta razón, es que a lo largo de este artículo se ha abogado por la necesidad de buscar la complementariedad y dotar de relevancia el concepto de noviolencia, dado que, para el caso nasa, si se habla de una mera resistencia civil, se está dejando de lado la importancia que tienen sus sueños o fines, desde la armonía, el equilibrio y el bienestar. Se estaría dejando de lado esa importante relación que han forjado las comunidades desde su vivencia, entre los medios y los fines.

De igual forma que la noviolencia ha querido distinguirse de simples métodos o tácticas (noviolencia), la resistencia que realizan los nasas también ha ido superado esa faceta pragmática (resistencia civil). La resistencia nasa se ha convertido en una resistencia civil noviolenta, en la que existe una relación entre medios y fines, en la que se construyen planes constructivos o planes de vida, en la que existen estrategias de defensa noviolenta, como la guardia indígena, ${ }^{17} \mathrm{y}$ en la que se piensa en la autonomía y la autodeterminación de los pueblos como forma de vida y no en la toma del poder o cambio de gobierno. La defensa de la vida como mandato dentro de las comunidades deja implícita la convicción y la idea de noviolencia en sus comunidades.

Es más, si se parte del concepto de resistencia civil noviolenta, para el caso concreto de las comunidades indígenas, de igual forma caeríamos en un equívoco en la medida en que lo "civil" en gran medida se escapa de la realidad de estas poblaciones, porque está pensado principalmente en contextos y circunstancias propiamente urbanos, en los que existen unas formas de movilización diferente, y principalmente donde sus motivaciones o reivindicaciones van encaminadas exclusivamente hacia la consecución de derechos y libertades prestadas en el marco de los Estados sociales de derecho, y no como en el caso de los nasas en unas luchas anticoloniales, en las que se busca la autodeterminación y la pervivencia como pueblos. Por esta razón, es conveniente referirse a una resistencia indígena noviolenta, en las que se tienen en cuenta diversos aspectos, como la acción colectiva, cooperativa o de un grupo o grupos de personas, sin armas, que, a través de diferentes medios, expresiones y acciones, piensan conscientemente en unos fines y que trabajan desde el cuidado de los medios.

\footnotetext{
${ }^{17}$ Las formas de defensa son importantes dado que en la resistencia civil, en muchas ocasiones, se da cabida al uso combinado entre acciones civiles con acciones militares o estatales, o en otros casos puede llegar a constituirse en el preámbulo de una lucha armada, cosa que va en detrimento e incongruencia con los postulados de una noviolencia específica o positiva.
} 
Esta definición se ha planteado detalladamente en la investigación de la tesis doctoral, y se constituye en una propuesta conceptual en el estudio de las comunidades indígenas, no solo en Colombia, sino en otras latitudes, donde existan dichas características.

\section{Conclusiones}

Para el presente trabajo, podemos encontrar seis conclusiones con las que se pretende comprometer sus ideas centrales y dejar sobre la mesa una discusión que se pueda tratar en otros estudios.

En primer lugar, a causa del paradigma de la violencia, se ha desdibujado la importancia de lo no armado, y eso ha llevado al uso indistinto de términos como resistencia pasiva, utópica o ineficaz. La mayoría de los estudios se han centrado en las resistencias armadas y no han dejado ver lo fundamentales y trascendentales que han sido las luchas no armadas.

Aunque las formas de resistencia han sido muy diversas y antiguas, el uso o no de las armas ha marcado una distinción y un factor relevante para distinguir las resistencias. La noción de resistencia por sí sola es muy genérica y, en esa medida, ha tenido que estar acompañada (incluso la Real Academia Española le añade el término pasiva). El término civil, por su parte, también ha sido otra de las acepciones que se ha incluido con este mismo propósito: darle una connotación no armada o la especificación de que no se trata de cuerpos militares o religiosos.

En segundo lugar, las concepciones de noviolencia complementan los conceptos de resistencia civil y desobediencia civil y ayudan a redimensionarlos. Los valores de la noviolencia permiten que el concepto de resistencia civil supere su mera dimensión pragmática y su connotación de simple acompañamiento de las luchas armadas. La noviolencia posibilita ver los lazos más profundos, como la autonomía o la cohesión social.

En tercer lugar, se pueden analizar casos, hechos y circunstancias reales a partir de la teoría de la noviolencia, por ejemplo la población indígena nasa. Diferentes autores en Colombia han partido de la noviolencia para llevar a cabo distintas investigaciones, entre ellas, la de Cante (2005), Ballesteros (2005) y Useche (2008), en las que se parte de la noviolencia como sustrato y fundamento para el estudio de casos y hechos particulares.

En cuarto lugar, se puede afirmar que la noviolencia, al tratarse de un recurso que tiene todo individuo antes de poder acceder a las armas, de un poder que se encuentra inmerso en cada persona, de un poder intrínseco que tiene cualquier comunidad, se manifiesta y es susceptible de lectura y de comparación en diferentes contextos y lugares del mundo. Aunque existan sustratos y valores culturales y cosmológicos diferentes, hay condiciones de resistencia, de rebeldía, de inconformidad, que dan cuenta de lo nocivo de la violencia y lo simplista que puede ser la pasividad, que llevan a conclusiones similares sintetizadas en gran medida en el concepto y los supuestos de la noviolencia. 
La noviolencia tiene la convicción de que es más revolucionario y más sedimentado el cambio y la transformación de los conflictos sin el recurso de las armas que con su uso.

En quinto lugar, se puede reafirmar que existe una noviolencia pragmática en la que no se tienen en cuenta los valores o la ideología; y aunque esta noviolencia pragmática, en cierto sentido, permite el acercamiento y una práctica inicial de la acción política noviolenta, está claro, como bien afirman Pontara o Gandhi, que es necesaria la congruencia y el cuidado tanto de los medios como de los fines. No son cosas que se puedan pensar por separado; la noviolencia no es compatible con cualquier ideología. La historia nos ha venido mostrando que ni todo fin justifica el uso de unos medios ni que los medios son absoluto garante de que los fines no se puedan llegar a pervertir.

No se puede pensar solo en la eficacia y eficiencia a la hora de obtener resultados, sino que se tienen que tener claros cuáles son los resultados que se están esperando (los fines). Experiencias como el nazismo en su primera fase dieron cuenta de ello y sirven como ejemplo en la medida en que usaron muchos de los métodos de persuasión y protesta que ha tratado Sharp (1973) como métodos de acción noviolenta. La eficacia de los medios no armados fue innegable y sirvió como estrategia para llegar a otros medios, como las armas, la coerción y la violencia legal.

El hecho de que las comunidades indígenas piensen en la armonía y el bienestar de sus comunidades desde todo lo que engloba la idea de kweet fxindxii -el buen vivir en nasa yuwe- hacen que los medios y los fines tengan gran compatibilidad, asunto que facilita la contrastación de la teoría noviolenta con la práctica propia y específica nasa. De esta forma, aunque de manera inicial el uso de la noviolencia pragmática permitió el acercamiento de las comunidades con la teoría, han sido los fines los que han provocado la permanencia de estas prácticas en sus formas de vida.

En sexto lugar, y con el propósito de dejar la discusión abierta, se podría preguntar sobre lo siguiente: ¿cuáles son los alcances y los límites de la teoría noviolenta? ¿Qué dificultades se encuentran para el análisis? ¿Cómo se puede retroalimentar?, es decir, ¿qué puede enseñar a los casos y hechos de la vida cotidiana y qué puede aprender de ellos? Estas son algunas de las preguntas que pueden quedar en el tintero, y que se intentarán resolver en posteriores investigaciones, al igual que con la continua discusión sobre el tema.

\section{Referencias}

Arendt, H. (2005a). ¿Qué es la política? Buenos Aires: Paidós.

Arendt, H. (2005b). Sobre la violencia. Madrid: Alianza.

Arias, G. (1995). Proyecto político de la noviolencia. Madrid: Nueva Utopía.

Aristófanes. Lisístratra. Recuperado de http://historicodigital.com/download/Aristofanes\%20-\%20Lisistrata.pdf 
Ballesteros Peluffo, G. L. (2005). Aspectos teóricos e históricos de la desobediencia civil. Papel Político, 18, 189-220.

Bhabha, H. K. (1994). El lugar de la cultura. Buenos Aires: Manantial.

Bravo, A. y Bruzzone, A. M. (1995). In guerra senza armi: storie di donne 1940-1945. Roma: Laterza.

Cante, F. (2005). Acción política noviolenta, una opción para Colombia. Bogotá: Universidad del Rosario.

de La Boétie, É. (1986). Discurso de la servidumbre voluntaria o el contra uno. Madrid: Tecnos.

Fanon, F. (2009). Piel negra, máscaras blancas. Buenos Aires: Akal.

Fisas, V. (2004). Procesos de paz y negociación en conflictos armados. Barcelona: Planeta.

Galtung, J. (1975). Violencia. En D. L. Sills (dir.), Enciclopedia internacional de ciencias sociales. Madrid: Aguilar.

Galtung, J. (1985). Sobre la paz. Barcelona: Fontamara.

Galtung, J. (2003). Paz por medios pacíficos: paz, conflicto, desarrollo y civilización. Bilbao: Gernika Gogoratuz.

Gandhi, M. (1998). Todos los hombres son hermanos. Madrid: Librería Dersa.

Gandhi, M. (2004). Escritos esenciales. Santander: Sal Terrae.

Gandhi, M. (2010). Política de la noviolencia. Madrid: Catarata.

Godwin, W. (1793). An enquiry concerning political justice and its influence on general virtue and happiness. Londres: GGJ and J. Robinson.

González Piñeros, N. C. (2006). Resistencia indígena: alternativa en medio del conflicto colombiano. Cali, Colombia: Pontificia Universidad Javeriana.

Guha, R. (2002). Las voces de la historia y otros estudios subalternos. Barcelona: Crítica.

Gramsci, A. (1934) Al margen de la historia. (Historia de los grupos sociales subalternos). Cuadernos de la Cárcel.

Habermas, J. (2000). La desobediencia civil. Piedra de toque del Estado democrático de derecho y derecho y violencia. Un trauma alemán. Barcelona: Península.

Hernández Delgado, E. (2004). Resistencia civil artesana de paz: experiencias indígenas, afrodescendientes y campesinas. Bogotá: Pontificia Universidad Javeriana. 
Harto de Vera, F. (2004). Investigación para la paz y resolución de conflictos. Valencia: Tirant lo Blanch.

Herranz Castillo, R. (1992). Henry D. Thoreau: resistencia y desobediencia civil. Madrid: Centro de Enseñanza Superior Luis Vives.

Houtart, F. (1999). La mundialización de las resistencias y de las luchas contra el neoliberalismo. París: L’Harmattan.

Houtart, F. (2006). Los movimientos sociales y la construcción de un nuevo sujeto histórico. La teoría marxista hoy: problemas y perspectivas. Buenos Aires: Consejo Latinoamericano de Ciencias Sociales.

Lederach, J. (2000). El abecé de la paz y los conflictos: educación para la paz. Madrid: Catarata.

López Martínez, M. (2004). Enciclopedia de paz y conflictos. Granada: Universidad de Granada.

López Martínez, M. (2006). Política sin violencia. Bogotá: Corporación Universitaria Minuto de Dios.

López Martínez, M. (2012a). Ni paz, ni guerra, sino todo lo contrario: ensayos sobre defensa y resistencia civil. Granada: Educatori.

López Martínez, M. (2012b). Gandhi, política y satyagraha. Ra-Ximhai, 8(2), 39-70.

López Martínez, M. (2013). Política sin matar: los métodos de la acción noviolenta. Vectores de Investigación, 7, 33-84.

Martínez Bernal, D. R. (2016). Caminando hacia la satyagraha: comunidad, lucha y kweet fxindxi en los NASA (1971-2015) (Tesis de doctorado, Universidad de Granada, Granada, España).

Merton, T. (2000). Gandhi y la noviolencia. Barcelona: Paidós.

Muñoz, F. A. (2001), La paz imperfecta. Granada, España: Universidad de Granada.

Negri, A. (2008). El movimiento de los movimientos: nuevas condiciones para el nuevo movimiento de los movimientos. Cuadernos del Pensamiento Crítico Latinoamericano, 15 .

Pontara, G. (1991). Noviolencia. En N. Bobbio, N. Matteuccci y G. Pasquino (eds.), Diccionario de política. Madrid: Siglo XXI.

Randle, M. (1998). Resistencia civil: la ciudadanía ante las arbitrariedades de los gobiernos. Barcelona: Paidós. 
Real Academia Española. Resistencia. Recuperado de http://dle.rae.es/?id=WAPyoek Said, E. (1990). Orientalismo. Madrid: Libertarias-prodhufi.

Scott, J. C. (2000). Los dominados y el arte de la resistencia. México: Era.

Sémelin, J. (1993). Unarmed against Hitler: Civilian resistance in Europe, 1939-1943. Westport: Praeger.

Seoane, J. y Taddei, E. (comps.) (2001). Resistencias mundiales: de Seattle a Porto Alegre. Buenos Aires: Consejo Latinoamericano de Ciencias Sociales.

Sharp, G. (1973). The politics of nonviolent action. Boston: Porter Sargent.

Shelley, P. B (1832). The masque of anarchy. Londres: Edward Noxon.

Sófocles (2001). Antígona. Santiago de Chile: Pehuen Editores. Recuperado de http:// www.colombiaaprende.edu.co/html/mediateca/1607/articles-65465_archivo.pdf

Spivak, G. C. (1997). ¿Puede hablar el sujeto subalterno? Buenos Aires: Orbis Tertius.

Touraine A. (1995). Producción de la sociedad. México: Universidad Nacional Autónoma de México.

Thoreau, A. (2002). Sobre el deber de la desobediencia civil (trad. A. Casado da Rocha). San Sebastián: Iralka.

Tilly, C. (2005). La democratización mediante la lucha. Sociológica, 57, 35-59.

Useche Aldana, O. (2008). La noviolencia: resignificación los derechos humanos. Polisemia, 5, 64-72.

Zibechi, R. (2003). Los movimientos sociales latinoamericanos: tendencias y desafíos. Osal, 9, 185-188. 
